\title{
Anode Fall Formation in a Hall Thruster
}

\author{
Leonid A. Dorf, Yevgeny F. Raitses, Artem N. Smirnov, and Nathaniel J. Fisch \\ Princeton Plasma Physics Laboratory, Princeton, New Jersey, 08543
}

\begin{abstract}
As was reported in our previous work, accurate, non-disturbing near-anode measurements of the plasma density, electron temperature, and plasma potential performed with biased and emissive probes allowed the first experimental identification of both electron-repelling (negative anode fall) and electron-attracting (positive anode fall) anode sheaths in Hall thrusters. An interesting new phenomenon revealed by the probe measurements is that the anode fall changes from positive to negative upon removal of the dielectric coating, which appears on the anode surface during the course of Hall thruster operation. As reported in the present work, EDS analysis of the chemical composition of the anode dielectric coating indicates that the coating layer consists essentially of an oxide of the anode material (stainless steel). However, it is still unclear how oxygen gets into the thruster channel. Most importantly, possible mechanisms of anode fall formation in a Hall thruster with a clean and a coated anodes are analyzed in this work; practical implication of understanding the general structure of the electron-attracting anode sheath in the case of a coated anode is also discussed.
\end{abstract}

\section{Introduction}

$I^{1}$ $\mathrm{N}$ a gas discharge, there can be either an increase or a drop in the plasma potential toward the anode, generally referred to in the literature as the "anode fall". When the anode is at a higher potential than the near-anode plasma, the anode fall is called "positive", and when it is at a lower potential - "negative". The positive and negative anode falls are essentially associated with formation of the electron-attracting and electron-repelling anode sheaths, respectively. The anode sheath is a thin space-charge layer adjoint to the electrode. It is a non-linear structure that was first observed and studied by Langmuir and Mott-Smith in glow discharges. ${ }^{1}$

In spite of a number of experimental ${ }^{2-12}$ and theoretical ${ }^{13-16}$ studies of a Hall thruster (HT) internal plasma structure, the understanding of the anode sheath phenomena in Hall Thrusters was, until recently, very limited. A more detailed review of previous works and motivation for studying the anode sheath phenomena in Hall Thrusters can be found in Refs. 17 and 18. As was reported in our recent publications, a diagnostic apparatus comprising biased and emissive electrostatic probes, a high-precision positioning system, and low-noise electronic circuitry was developed and used for measurements in the near-anode region of the $12.3 \mathrm{~cm}$ Hall thruster operating in the $0.2-2$ $\mathrm{kW}$ power range ${ }^{17,19}$. Accurate, non-disturbing near-anode measurements of the plasma density, electron temperature, and plasma potential performed with biased and emissive probes allowed the first experimental identification of both electron-repelling (negative anode fall) and electron-attracting (positive anode fall) anode sheaths in Hall thrusters. Most interestingly, an intricate new phenomenon revealed by the probe measurements is that the anode fall changes from positive to negative upon removal of the dielectric coating, which appears on the anode surface during the course of Hall thruster operation. The dependence of the sign and magnitude of the anode sheath on the discharge voltage and mass flow rate was also studied in Ref. 17, and the results of this investigation were found to be in agreement with our recent theoretical model. ${ }^{17,18,20}$ Particularly, it was found that when the electron temperature, $T_{e}$, in the channel increases with the discharge voltage, $V_{d}$, the magnitude of the negative anode fall at a clean anode also increases with $V_{d}$.

In this paper, we report our most recent findings about the nature of the anode dielectric coating, present pictures of the clean and coated anodes, as well as the visual evidence of $2 \mathrm{~kW}$ Hall thruster operation in both cases, and analyze possible mechanisms of anode fall formation in a Hall thruster with the clean and coated anodes; we conclude with discussing possible practical implications of the presented work. 


\section{Anode Dielectric Coating}

\section{A. Coating Formation and Appearance}

It was observed experimentally for the $2 \mathrm{~kW}$ Hall thruster in this study that a dielectric coating appears on the anode surface exposed to plasma after the thruster accumulates about 10 or more hours of lifetime, operating at typical conditions (Fig. 1). A layer of coating was typically thin - about several tens of microns - and ranged in color from blue-green to golden-brown, with sometimes several colors present on the same coated anode. In some cases, after several tens of hours of thruster operation, the coating would accumulate to be up to $0.1 \mathrm{~mm}$ thick, and then it would split off - the scraps of coating were found at the bottom part of the channel. Furthermore, distinctive spots were observed next to each of the gas-injecting holes hidden under the gas-mixing baffles (Fig. 1).
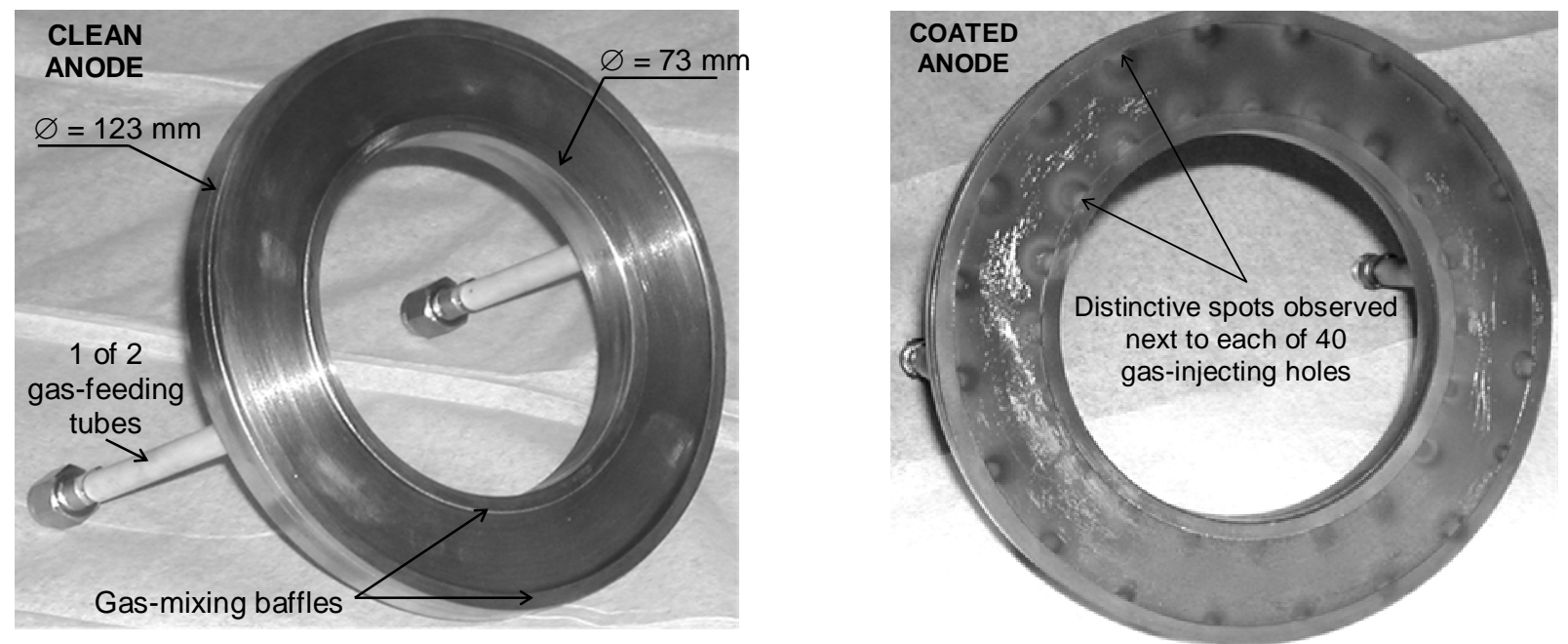

Figure 1: Anode of the $\mathbf{2} \mathrm{kW}$ Hall thruster before (left) and after (right) thruster operation. Accumulated lifetime $>10$ hours.

\section{B. Coating Chemical Composition}

To identify the chemical composition of the coating, three small samples were inserted under the baffle at the bottom part of the anode. Two samples were made out of the same material as the anode - stainless steel - and one sample was made out of tantalum. After several hours of thruster operation, the samples were extracted and a dielectric coating was observed on each of the samples. The coating on the tantalum sample was of a homogeneous blue-green color, and the coating on the stainless steel samples was of a yellow-red color. All three samples were then sent to Stork MMA Testing Laboratories (Newtown, PA) for Energy Dispersion Spectroscopy (EDS) analysis of the coating material. Results of EDS analysis - counts per energy spectra of the X-ray radiation emitted by the atoms of the coating as a result of electron bombardment - are reported in Ref. 18. It was ultimately suggested by Stork MMA metallurgists that the coatings on all three samples are essentially the oxides of the substrate materials. The anode material, stainless steel, can indeed be expected to get oxidized during thruster operation, since the anode temperature could reach about $1000 \mathrm{C}$ at typical thruster operating conditions.

It is still unclear, however, how oxygen gets into the thruster channel. Impurity limits data for a research grade Xenon used in the presented experiments (see Ref. 18) suggests that only insignificant amount of oxygen enters the channel along with the xenon propellant, since the percentage of $\mathrm{O}_{2}$ in the cylinder containing the supply of xenon is very low - less than $0.1 \mathrm{ppm}$ (parts per million). It can alternatively be suggested that the presence of oxygen in the thruster channel is the vacuum facility effect - ougassing of the vacuum vessel walls and cryogenic pumps interior could be one of the sources of oxygen, for example. A detailed investigation of oxygen origination and its content in the vacuum vessel needs to be conducted with the use of a residual gas analyzer (RGA) that is already installed on one of the ports of the HTX vacuum vessel. Information about the content of oxygen in the background gas inside the vacuum vessel can then be used to estimate the anode surface oxidation rate and coating formation time. 


\section{Operation of the $\mathbf{2} \mathbf{k W}$ Hall Thruster with Clean and Coated Anodes}

Figure 2 shows discharge current, $I_{d}$, vs. discharge voltage, $V_{d}$, characteristics of the $12.3 \mathrm{~cm}$ Hall thruster with the clean and coated anodes (referred to as "Clean" and "Coat", respectively) for several mass flow rates. As can be seen, the characteristics are similar for two anodes. Figure 3 shows some of the results of biased probe measurements in the near-anode region of the $2 \mathrm{~kW}$ Hall thruster with the clean and coated anodes, respectively, for $\dot{m}=5 \mathrm{mg} / \mathrm{s}$. Zero potential and $Z=0$ are chosen at the anode. As can be seen from Fig. 3, in the case of the clean anode, the plasma potential at $2-12 \mathrm{~mm}$ from the anode is higher than the anode potential - the anode fall is negative. This indicates the presence of an electron-repelling anode sheath predicted theoretically in Hall thrusters. ${ }^{13,16,20}$ Alternatively, in the case of the coated anode, the plasma potential at $2-12 \mathrm{~mm}$ from the anode is lower than the anode potential - the anode fall is positive. This indicates the presence of an electron-attracting anode sheath. Furthermore, it was observed that thruster operation with a coated anode is associated with a visual effect: the gas-injecting holes glow brighter than the rest of the anode, with appearance of a jet-like structure from each hole (Fig. 4).

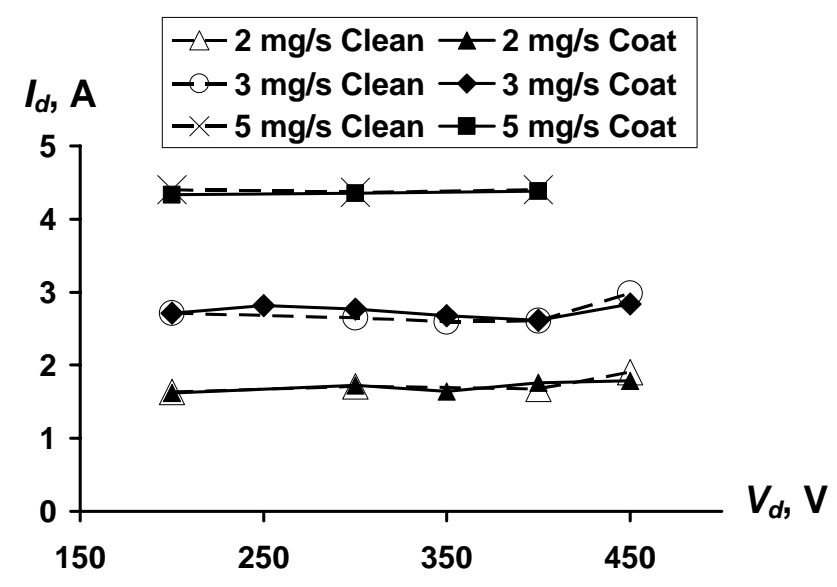

Figure 2: Discharge current, $I_{d}$, vs. discharge voltage, $V_{d}$, characteristics of the $12.3 \mathrm{~cm} \mathrm{HT}$. Measured at several mass flow rates for the clean and coated anodes.

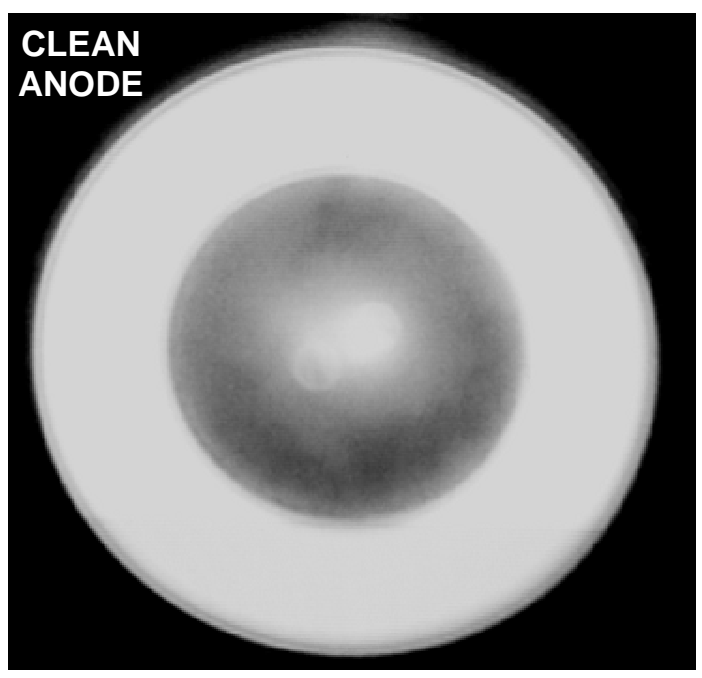

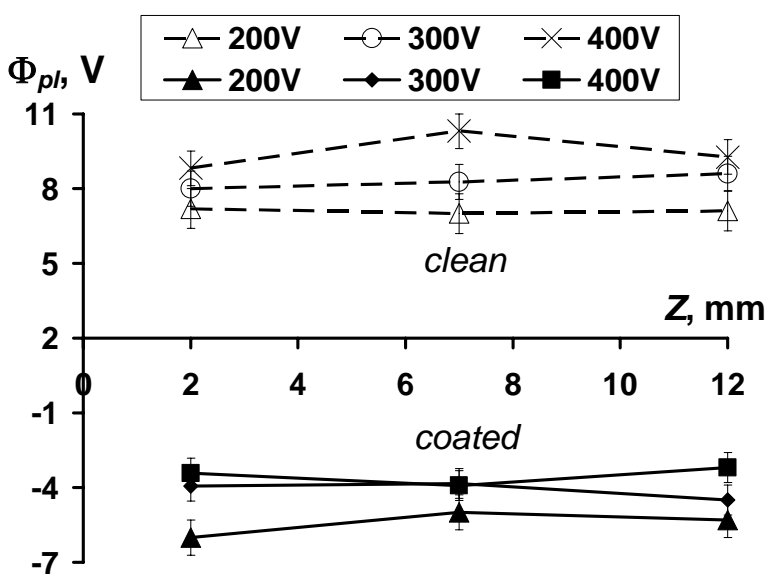

Figure 3: Results of biased probe measurements in the near-anode region of the $12.3 \mathrm{~cm} \mathrm{HT}$.

Plasma potential axial profile, $\Phi_{p l}(z)$, for the clean and coated anodes measured at the mass flow rate $\dot{m}=5 \mathrm{mg} / \mathrm{s}$ and several discharge voltages, in the midpoint between the channel walls $(R=49 \mathrm{~mm}$ from the thruster axis).

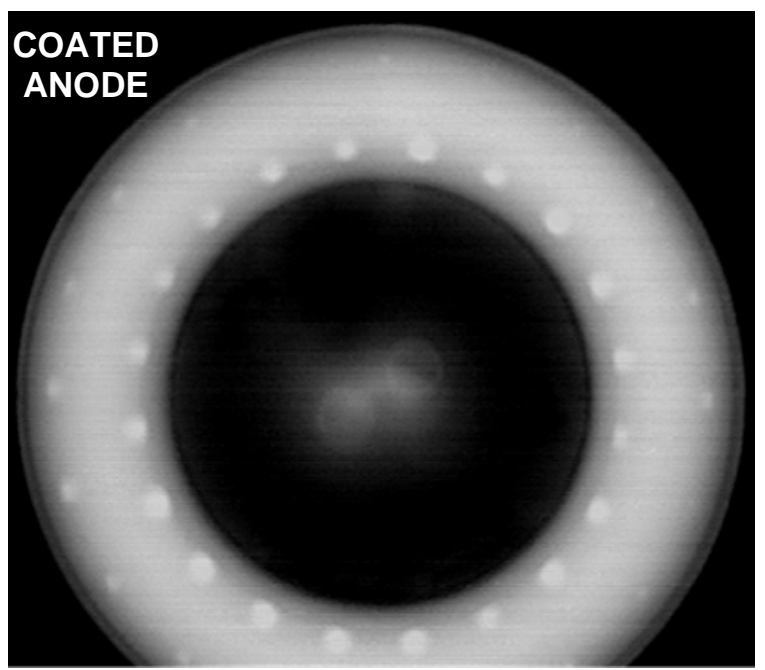

Figure 4: Photographs of $2 \mathrm{~kW}$ HT operation with the clean and coated anodes. 


\section{Possible Mechanisms of Anode Fall Formation}

\section{A. Neutral Density Distribution}

To understand anode sheath formation, first it is useful to analyze the distribution of the neutral density near and inside the anode. For $\dot{m}=5 \mathrm{mg} / \mathrm{s}$, rough density estimations yield that mean free path for neutral-neutral collisions inside the anode and gas-injecting holes is smaller than the diameter of the hole (Fig. 5), so hydrodynamic Bernoulli equation for neutral pressure, $P_{n}$, neutral density, $n_{n}$, and neutral flow velocity, $V_{n}$, can be employed to relate flows inside the holes and inside the anode: $P_{n}+M_{n} n_{n} V_{n}^{2} / 2=$ Const,

where $M_{n}=2.2 \cdot 10^{-22} \mathrm{~g}$ is the mass of a Xenon atom. ${ }^{22}$ Taking into account the flow continuity equation along with the fact that the cross-sectional area of the anode cavity (almost equal to the collecting surface area of the clean anode, $A_{\text {coll }}=77 \mathrm{~cm}^{2}$ ) is much larger than the combined cross-sectional area of the holes, $A_{H}=0.03 \mathrm{~cm}^{2}$, the dynamic pressure term can be neglected inside the anode. Similar to the problem of the flow of gas through the nozzle, ${ }^{22}$ the neutral flow is assumed to have a sonic velocity inside the holes. For the isothermal flow with the neutral temperature equal to the anode temperature, $T_{A} \approx 1000 \mathrm{C}$, using the anode dimensions given in Fig. 5, it can be finally

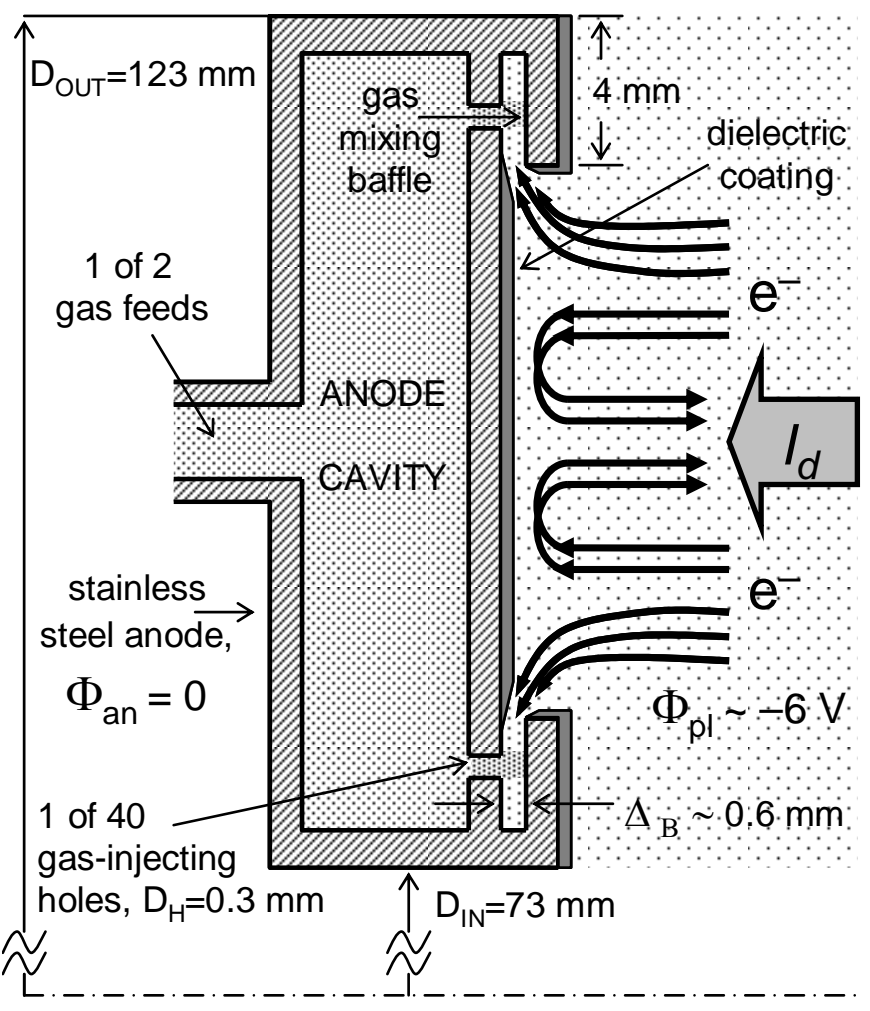

Figure 5: Possible mechanism of formation of a positive fall at the coated anode estimated for $\dot{m}=5 \mathrm{mg} / \mathrm{s}$ that: (1) - the neutral density inside the holes is $n_{H}=\dot{m} /\left(M_{n} \cdot A_{H} \cdot \sqrt{T_{A} / M_{n}}\right)=2.7 \cdot 10^{16} \mathrm{~cm}^{-3}$, (2) - the static pressure in the holes is $P_{H}=n_{H} T_{a} \approx 3$ Torr , and it is twice as big as the dynamic pressure, (3) - the static pressure inside the anode is $P_{A}=3 / 2 P_{H} \approx 4.5$ Torr, and (4) - the neutral density inside the anode is $n_{A} \approx 4 \cdot 10^{16} \mathrm{~cm}^{-3}$. For the neutralneutral collision cross-section $\sigma_{n n} \approx 3 \cdot 10^{-15} \mathrm{~cm}^{2}$, the length of the mean free path inside the holes can be estimated as $\Lambda_{H}=1 /\left(\sigma_{n n} \cdot n_{H}\right) \approx 0.12 \mathrm{~mm}$, which is smaller than the hole diameter, $D_{H}=0.3 \mathrm{~mm}$. This estimation supports the aforementioned assumption of the hydrodynamic flow inside the holes. Assume now that after the jets that are coming out of the gas-injecting holes hit the baffles (Fig. 5), the neutral gas spreads out and homogeneously fills up the volume under the baffles. Then, assuming that the neutral gas leaves the volume under the baffles and enters the channel with the velocity of a free atomic flow, $V_{a}=\sqrt{T_{A} /\left(2 \cdot \pi \cdot M_{n}\right)} \approx 113 \mathrm{~m} / \mathrm{s}$, the average neutral density, neutral pressure, and the length of the mean free path for neutral-neutral collisions under the baffles can be estimated as $n_{B} \approx \dot{m} /\left(M_{n} \cdot V_{a} \cdot A_{B}\right) \approx 5.5 \cdot 10^{14} \mathrm{~cm}^{-3}, P_{B}=n_{B} T_{A} \approx 75 \mathrm{mTorr}$, and $\Lambda_{B}=1 /\left(\sigma_{n n} \cdot n_{B}\right) \approx 6 \mathrm{~mm}$, respectively. In the above estimation, $A_{B}=\pi \cdot\left(D_{\text {out }}+D_{\text {in }}\right) \cdot \Delta_{B} \approx 3.7 \mathrm{~cm}^{2}$ is the area of the surface through which the gas leaves the volume under the baffles, calculated using the dimensions given in Fig. 5. Finally, when the neutral gas distributes uniformly over the entire channel cross-section (see Ref. 23) and bleeds through the channel with the velocity $V_{a}$, the density, pressure, and the length of the mean free path near the anode can be estimated as $n_{C} \approx \dot{m} /\left(M_{n} \cdot V_{a} \cdot A_{\text {coll }}\right) \approx 2.6 \cdot 10^{13} \mathrm{~cm}^{-3}, P_{C}=n_{C} T_{A} \approx 3.5 \mathrm{mTorr}$, and $\Lambda_{C}=1 /\left(\sigma_{n n} \cdot n_{C}\right) \approx 12 \mathrm{~cm}$, respectively. 


\section{B. Formation of a Negative Fall at the Clean Anode}

In the quasineutral plasma between the clean anode and the acceleration region, the electron drift velocity, $V_{d r} \sim I_{d} /\left(e \cdot n \cdot A_{\text {coll }}\right) \approx 10^{7} \mathrm{~cm} / \mathrm{s}$, is much smaller than the average velocity of the half-Maxwellian electron flux that would be traveling toward the anode in the absence of an electron-repelling anode sheath, $V_{H M}=\sqrt{2 T_{e} /\left(\pi m_{e}\right)} \approx 8 \cdot 10^{7} \mathrm{~cm} / \mathrm{s}$. The above estimation was made using the channel cross-section equal to $A_{\text {coll }}=77 \mathrm{~cm}^{2}$, the measured discharged current, $I_{d}=4.35 \mathrm{~A}$, and the plasma density, $n$, along with the electron temperature, $T_{e}$, measured in the mid-point between the channel walls at $2 \mathrm{~mm}$ from the anode for $V_{d}=200 \mathrm{~V}$ and $\dot{m}=5 \mathrm{mg} / \mathrm{s}$ (this data is presented in Refs. 18 and 21). Thus, the thermal electron flux to the anode would carry an electric current much greater than the discharge current. At mass flow rates typical for the $2 \mathrm{~kW}$ Hall thruster, electron-neutral collisions are very weak near the anode $\left(\Lambda_{e n} \sim \Lambda_{n n} \approx 12 \mathrm{~cm}\right)$, so is the magnetic field in the conventional configuration (see Ref. 18). Therefore, formation of an electron-repelling anode sheath (negative anode fall) is required to repel an excessive electron flux from the anode. A reversed electron flux created in the electronrepelling anode sheath decreases the net electron velocity in the quasineutral plasma near the anode to the value $V_{d r}$ determined by the discharge current, thus providing current continuity everywhere.

\section{Formation of a Positive Fall at the Coated Anode}

In the case of the coated anode (Fig. 5), the electron drift velocity estimated the same way as above, is even smaller, $V_{d r} \approx 5 \cdot 10^{6} \mathrm{~cm} / \mathrm{s}$, because for the same operating conditions the plasma density measured near the coated anode is almost twice as big as it is near the clean anode (see Refs. 17 and 18). Since the electron thermal velocity is almost the same (see Refs. 17 and 18), formation of an electron-repelling sheath at the coated anode surface is again required to decrease the net electron velocity in the quasineutral plasma near the anode from $V_{H M}$ to $V_{d r}$. By reflecting electrons and creating an ion backflow this sheath also allows satisfying the condition of a zero net current to dielectric.

\section{Electron-Attracting Anode Sheath}

While the anode surface exposed to plasma gets coated with dielectric, some elements of the anode surface, for example the inner side of the gas-mixing baffles or the interior of the gas-injecting holes, may remain conductive (Fig. 5). When the anode front surface is coated with dielectric, the discharge current supposedly closes to the anode at these conductive surfaces by the electrons that are not repelled. A half-Maxwellian electron flux entering the volume under the baffles carries the electric current $I_{H M}=1 / 2 \cdot(1.5 \cdot n) \cdot \sqrt{2 T_{e} /\left(\pi m_{e}\right)} \cdot e \cdot A_{B} \approx 1.76 \mathrm{~A}$, which constitutes about $40 \%$ of the measured discharge current, $I_{d}=4.32 \mathrm{~A}$ (see Figs. 2 and 5 ). The above estimation is made using the plasma density, $n$, and electron temperature, $T_{e}$, measured in the mid-point between the channel walls at $2 \mathrm{~mm}$ from the anode for $V_{d}=200 \mathrm{~V}$ and $\dot{m}=5 \mathrm{mg} / \mathrm{s}$ (see Refs. 18 and 21), and considering the maximum ( $\sim 50 \%)$ relative error in determining the plasma density from biased probe measurements (see Ref. 19). The remainder $60 \%$ of the electron flux gets drawn in under the baffles by the electron-attracting sheath that appears at the inner, metal sides of the baffles. Due to this sheath, the near-anode plasma potential appears to be about $4-6 \mathrm{~V}$ lower than the anode potential (Fig. 3). The plasma in this electron-attracting sheath can be almost purely electron, like in a vacuum diode, since its characteristic scale, $\Delta_{B}=0.6 \mathrm{~mm}$ (Fig. 5), is only several Debye lengths estimated at $2 \mathrm{~mm}$ from the anode, $\lambda_{D}=\sqrt{T_{e} /\left(4 \pi \cdot n_{e} \cdot e^{2}\right)} \sim 0.075 \mathrm{~mm}$. The electron density under the baffles in the case of the coated anode is much larger than in the case of the clean anode, in which only the electrons that penetrate through the electron-repelling sheath enter the volume under the baffles. This could explain such intricate phenomena as distinctive glowing next to each of the gas-injecting holes (where the neutral density is the largest) observed during operation of the $12.3 \mathrm{~cm}$ Hall thruster with the coated anode (Fig. 4).

\section{Enhanced Ionization}

It was observed experimentally for Hall thrusters that penetration of the electrons into the anode cavity can cause ionization of the neutral gas inside the anode. ${ }^{3}$ Furthermore, near the anode, the discharge of a Hall thruster with a coated anode can be compared to that of an extensively studied hollow-anode plasma source ${ }^{24,25}$, as the coated anode has two essential features of the hollow anode: a collecting surface area that is significantly smaller than the cross-sectional area of the discharge chamber, and an insulated front surface. A high degree of ionization inside the hollow-anode plasma source - near-anode electron density of $10^{10}-10^{11} \mathrm{~cm}^{-3}$ at nitrogen flow rate of $25 \mathrm{scc} / \mathrm{min}$ - was attributed to formation of a thick electron-attracting anode sheath, in which electrons gain kinetic 
energy of up to $40 \mathrm{eV} .^{26}$ Thus, it can be suggested that formation of a positive fall at the coated anode in the $2 \mathrm{~kW}$ Hall thruster could be explained by the need for enhanced ionization: additional ionization in the sheath or inside the anode would increase the electron flux toward anode conductive surfaces to the value determined by the discharge current. ${ }^{17}$ However, the neutral gas pressure in front of the anode in a hollow-anode plasma source is about 100 times larger (and therefore the ionization length is about 100 times smaller) than it is near the coated anode in a Hall thruster (see Ref. 26). Furthermore, as it was later reported by Melikov in Ref. 4, noticeable ionization inside the anode occurs only if the anode temperature is lower than $500 \mathrm{C}$. At higher temperatures, the neutral pressure, $p_{n} \geq 2$ Torr, becomes comparable to the electron pressure, $p_{e} \sim 1.9$ Torr, and the discharge is carried by the gas

flow out of the anode cavity (these estimations were made for the plasma density $n=10^{10} \mathrm{~cm}^{-3}$, electron temperature $T_{e}=8 \mathrm{eV}$, and mass flow rate of $2 \mathrm{mg} / \mathrm{s}$ ). Also noteworthy is that spectroscopic measurements inside a hollow-anode plasma source performed by Miljevic (see Ref. 25) were later repeated by Young et al (see Ref. 27), who observed the ionization degree of only $0.06-0.15 \%$.

The following estimations can be used to demonstrate that formation of a positive anode fall at the coated anode in the $2 \mathrm{~kW}$ Hall thruster is unlikely to be explained by the need for enhanced ionization. For a half-Maxwellian electron flux with the temperature $T_{e B} \sim 10 \mathrm{eV}$ and neutral densities calculated above, ionization lengths in front of the anode, under the baffles, inside the gas-injecting holes, and inside the anode can be estimated as $\Lambda_{C}^{i} \sim 1 /\left(<\sigma^{i}>\cdot n_{C}\right) \approx 1 \mathrm{~m}, \quad \Lambda_{B}^{i} \sim 4.5 \mathrm{~cm}, \quad \Lambda_{H}^{i} \sim 1 \mathrm{~mm}, \quad$ and $\quad \Lambda_{A}^{i} \sim 0.6 \mathrm{~mm}, \quad$ respectively. Here, $\left.<\sigma^{i}\right\rangle=<\sigma^{i} \cdot V_{e}>/ V_{H M} \approx 4 \cdot 10^{-16} \mathrm{~cm}^{2}$ is the energy-dependent electron impact ionization cross-section for Xenon atoms, averaged over the half-Maxwellian distribution (see Refs. 18 and 28). Thus, significant multiplication of the electron flux entering the volume under the baffles could occur only inside the holes or inside the anode. While electrons produced by ionization would reach the metal surface and contribute to the discharge current, corresponding ions would have to stream out into the channel and recombine with electrons at the anode dielectric surface, to provide charge conservation and current continuity everywhere. As follows from the estimations above, the ion flux from the volume under the baffles would then represent about $60 \%$ of the discharge current, so ion density under the baffles can be estimated as $n_{i B} \sim 0.6 \cdot I_{d} /\left(e \cdot V_{i B} \cdot A_{B}\right) \approx 3.3 \cdot 10^{13} \mathrm{~cm}^{-3}$, assuming ions are accelerated in the positive anode fall to the velocity $V_{i B} \sim \sqrt{e \cdot \Phi_{p l} / M_{X e}} \approx 2 \cdot 10^{5} \mathrm{~cm} / \mathrm{s}$. The plasma density inside the holes would have to be even larger, since $A_{B} / A_{H} \sim 100$. However, the plasma densities measured inside the Hall thruster anode (Ref. 3), in the vicinity of the anode hole in a hollow-anode plasma source (Ref. 26), and at 2 $\mathrm{mm}$ from the anode in the $12.3 \mathrm{~cm}$ Hall thruster (Ref.18) were observed to be of the order of $10^{10}-10^{11} \mathrm{~cm}^{-3}$. Thus, additional ionization inside the anode and gas-injecting holes is unlikely to be the process that maintains current balance at the anode surface, from which we conclude that formation of a positive fall at the coated anode is unlikely to be explained by the need for enhanced ionization.

\section{Summary and Discussion}

The positive anode fall formation mechanism suggested in this work can be summarized as follows: (1) - when the anode front surface is coated with dielectric, the discharge current closes to the anode at the surfaces that remain conductive, (2) - a total thermal electron current toward the conductive area is significantly smaller than the discharge current, therefore an additional electron flux needs to be attracted toward the conductive surfaces by the electron-attracting sheath that appears at these surfaces. Note that understanding the anode sheath structure in the case of a coated anode might be useful for designing a thruster anode. It is yet unclear how oxygen that forms the anode dielectric coating (oxide layer) gets into the discharge chamber, but since the vacuum facility used in the presented experiments is typical for studies of a Hall thruster, it can be suggested that coating formation is a general issue for laboratory Hall Thrusters [see, for example, Ref. 29, pp. 214, 377]. Moreover, if oxygen enters the thruster channel along with the xenon propellant, this issue could also be expected for thrusters used in actual space applications. Therefore, when designing an anode (which is not necessarily also a gas distributor) for a HT, one has to ensure that some surfaces will remain conductive if the anode front surface gets coated with a dielectric, so that combined conductive surface area is sufficient for passing a discharge current. Furthermore, the electron energy flux toward these surfaces in the case of a coated anode will be much higher than in the case of a clean anode, in which only the electrons that penetrate through the electron-repelling sheath reach the anode; plus, electrons gain additional kinetic energy while moving inside the electron-attracting sheath toward the anode conductive surfaces. Thus, one also has to ensure that these surfaces will not overheat during thruster operation. 


\section{Acknowledgments}

The authors are grateful to Dr. Vladimir Semenov and David Staack for fruitful discussions. This work was supported by US DOE Contract No. AC02-76CH0-3073 and NJ Commission for Science and Technology.

\section{References}

1 Langmuir, I. and Motт-Smith H. M. (1924). Gen. Elec. Rev. 27, 449, 538, 616, 761, 810. Also see: The Collected Works of Irving Langmuir, edited by SuITs, C. G. and WAY, H. E. (Pergamon, New York, 1961), Vol. 4.

2 Morozov, A. I., Esinchuk, Yu. V., Tilinin, G. N., Trofimov, A. V., Sharov, Yu. A., and ShChePKin, G. Ya. (1972). Plasma Accelerator with Closed Electron Drift and Extended Acceleration Zone. Sov. Phys. Tech. Phys. 17, 38.

3 Melikov, I. V. (1974). Experimental Investigation of Anode Processes in a Closed electron-drift accelerator. Sov. Phys. Tech. Phys. 19, 35.

4 Melikov, I. V. (1977). Point and Jet Ionization in a Hall Plasma Accelerator. Sov. Phys. Tech. Phys. 22, 452.

5 Bishaev, A. M. and KIM, V. (1978). Local Plasma Properties in a Hall-Current Accelerator with an Extended Acceleration Zone. Sov. Phys. Tech. Phys. 23, 1055.

6 Guerrini, G., Michaut, C., Dudeck, M., Vesselovzorov, A. N., and Bacal, M. (1997). Characterization of Plasma Inside the SPT-50 Channel by Electrostatic Probes. Proceedings of the $25^{\text {th }}$ International Electric Propulsion Conference, Aug 1997, Cleveland, OH, IEPC Paper 1997-053.

7 Raitses, Y., Ashrenazy, J., and Guelman, M. (1998). Propellant Utilization in Hall Thrusters. J. Prop. Power $14,247$.

8 Raitses, Y., Dorf, L. A., Litvak, A. A., and Fisch, N. J. (2000). Plume Reduction in Segmented Electrode Hall Thruster. J. Appl. Phys. 88, 1263.

Raitses, Y., Keidar, M., StaAcK, D., Fisch, N. J. (2002). Effects of Segmented Electrode in Hall Current Plasma Thruster. J. Appl. Phys. 92, 4906.

10 HaAs, J. M. and Gallimore, A. D. (2001). Internal Plasma Potential Profiles in a Laboratory-Model Hall Thruster. Phys. Plasmas 8, 652.

11 Meezan, N. B., Hargus, W. A., Jr., and Cappelli, M. A. (2001). Anomalous Electron Mobility in a Coaxial Hall Discharge Plasma. Phys. Rev. E 63, 026410.

12 Warner, N. Z., Szabo, J. J., and Martinez-Sanchez, M. (2003). Characterization of a High Specific Impulse Hall Thruster Using Electrostatic Probes. Proceedings of the $28^{\text {th }}$ International Electric Propulsion Conference, March 2003, Toulouse, France, IEPC Paper 2003-082.

13 Ahedo, E., Martinez-Cerezo, P., and Martinez-Sanchez, M. (2001). One-Dimensional Model of a Plasma Flow in a Hall Thruster. Phys. Plasmas 8, 3058.

14 Fruchtman, A., Fisch, N. J., and Raitses, Y. (2001). Control of the Electric Field Profile in the Hall Thruster. Phys. Plasmas 8, 1048.

15 Keidar, M., Boyd, I., and Beilis, I. (2002). Analyses of the Anode Region in a Hall Thruster Channel. 38th Joint Propulsion Conference and Exhibit, July 2002, Indianapolis, IN, AIAA Paper 2002-4107.

16 Dorf, L., Semenov, V., Raitses, Y., and Fisch, N. J. (2002). Hall Thruster Modeling with a Given Temperature Profile. 38th Joint Propulsion Conference and Exhibit, July 2002, Indianapolis, Indiana, AIAA paper 2002-4246.

17 Dorf, L., Raitses, Y., Semenov, V., and Fisch, N. J. (2004). Effect of Anode Dielectric Coating on Hall Thruster Operation. Appl. Phys. Let. 84, 1070.

18 Dorf, L. (2004). Studies of Anode Sheath Phenomena in Hall Thrusters. Ph.D. Dissertation. Princeton University, Princeton, NJ 08544, USA.

19 Dorf, L., RAITSES, Y., and FISCH, N. J. (2004). Electrostatic Probe Apparatus for Measurements in the Near-Anode Region of Hall Thrusters. Rev. Sci. Instrum., 75, 1255.

20 Dorf, L., Semenov, V., and Raitses, Y. (2003). Anode Sheath in Hall Thrusters. Appl. Phys. Let. 83, 2551.

21 Dorf, L., Raitses, Y., and Fisch, N. J. (2004). Experimental Studies of Anode Sheath Phenomena in a Hall Thruster Discharge. Submitted to J. Appl. Phys.

22 Landau, L. D., and Lifshitz, E. M. (1987). Fluid Mechanics. In Course of Theoretical Physics, 2nd edition (ButterworthHeinemann, Oxford), Vol. 6.

23 Vial, V., Lazurenko, A., Laure, C, and Bouchoule, A. (2003). Xenon Gas Injection in SPT Thrusters. Proceedings of the $28^{\text {th }}$ International Electric Propulsion Conference, March 2003, Toulouse, France, IEPC Paper 2003-0221.

24 MilJEVIC, V. I. (1984). Hollow Anode Ion-Electron Source. Rev. Sci. Instrum. 55, 931.

25 Miljevic, V. I. (1984). Spectroscopy of Hollow Anode Discharge. Appl. Optics 23, 1598.

26 Anders, A. and AnDERs, S. (1995). The Working Principle of the Hollow-Anode Plasma Source. Plasma Sources Sci. Technol. 4, 571 (Printed in the UK).

27 Young, M., Muntz, E. P., and Ketsdever, A. D. (1998). Investigation of a Candidate Non-Magnetic Ion Micro-Thruster for Small Spacecraft Applications. 34 ${ }^{\text {th }}$ Joint Propulsion Conference and Exhibit, July 1998, Cleveland, OH, AIAA Paper 19983917.

28 Rapp, D. and Englander-Golden, P. (1965). Total Cross Sections for Ionization and Attachment in Gases by Electron Impact: I. Positive Ionization. J. Chem. Phys. 43, 1464.

29 Morozov, A. I., and Savelyev, V. V (2000). Fundamentals of Stationary Plasma Thruster Theory. In Reviews of Plasma Physics, edited by Kadomtsev, B. B. and Shafranov, V. D. (Kluwer Academic/Plenum Publishers, New York), Vol. 21. 